\title{
RESEARCH HIGHLIGHT New myotonic dystrophy type 1 mouse model
}

\author{
Yunping Lei ${ }^{1}$ and Richard H. Finnell ${ }^{1,2}$ \\ Cell Research (2020) 30:99-100; https://doi.org/10.1038/s41422-020-0276-y
}

\begin{abstract}
Myotonic dystrophy type 1 (DM1) is a genetic disorder which compromises multiple organs and for which investigators lack a suitable mouse model for mechanistic and potential drug screening studies. Yin and colleagues using the latest genetic editing method, the CRISPR-Cas9 technique, started from newly established androgenic haploid embryonic stem cells to make a triple heterozygous mutant DM1 mouse model, as well as a quadruple heterozygous mutant DM1 mouse model, which faithfully recapitulates the pathological phenotypes of human DM1.
\end{abstract}

Myotonic dystrophy (DM), the most common form of muscular dystrophy, is a complex autosomal dominant disease with a varied range of symptoms. The typical clinical presentation includes: myotonia, muscular dystrophy, gradually worsening muscle loss and weakness, posterior cataracts, and endocrine disruptions. ${ }^{1}$ There are two types of DM, type $1 \mathrm{DM}$ (DM1) is known to be caused by an expansion of a cytosine-thymine-guanine (CTG) trinucleotide repeat in the $3^{\prime}$-untranslated region (3'-UTR) of the myotonic dystrophy protein kinase (DMPK) gene, ${ }^{2,3}$ while myotonic dystrophy type 2 (DM2) is the result of cytosine-cytosinethymine-guanosine (CCTG) tetranucleotide expansion in the first intron of the cellular nucleic acid-binding protein (CNBP) gene, namely zinc finger protein 9 (ZNF9) gene. ${ }^{4}$ Despite clinical similarities, DM1 and DM2 are two distinct disorders, with DM1 being generally more severe than $\mathrm{DM} 2$, requiring additional studies to better characterize the differences between these clinical entities.

Although the causative genes for both types of myotonic dystrophy are known, at the present time researchers lack a suitable DM1 mouse model that accurately recapitulates all of the molecular and clinical phenotypes of this devastating disease. This is partly due to the complex mechanism underlying DM1. To date, three models were published that attempted to explain the molecular pathologies associated with DM1. First was the haploinsufficiency model, which is based on the observation that expansion of the CTG repeat in the DMPK 3'-UTR reduced both mRNA and protein levels of DMPK. ${ }^{5}$ Secondly, the RNA toxicity model, was established based on the understanding that nuclear accumulated RNA containing the mutant abnormally long CUG tandem repeats aberrantly recruits splicing regulators, including MBNL1, to form the ribonuclear aggregates (foci) and finally result in the disruption of normal RNA splicing. ${ }^{6}$ The last of the published DM1 mouse models is the chromatin structure malformation model, which was based on the rationale that there is decreased expression of its downstream gene SIX5/DMAHP $P^{7}$ and upstream gene $D M W D^{8}$ in DM1 patients and their derivative cells. These three models involved four genes, namely DMPK, SIX5, MBNL1 and $D M W D$, connected by the DMPK gene. Previously, scientist tried to create DM1 mouse models by knocking out three of the four genes individually, including $D m p k^{-1-}$, Six $5^{-1-}$ and $M b n / 1^{-1-}$, or overexpressing the human skeletal actin (HAS) gene, which carries 250 untranslated CUG repeats to mimic the CUG expansion status in DM1 patients. ${ }^{9}$ However, none of the mouse models developed the clinical phenotypes found in DM1 patients. ${ }^{9}$ Yin and colleagues utilized a novel approach testing whether mice carrying three or four of the key DM1 genes as heterozygous lossof-function (LoF) mutants, can better recapitulate the DM1 phenotypes using their recently established androgenic haploid embryonic stem cells (AG-haESCs) and the latest CRISPR-Cas9 genetic editing techniques (Fig. 1).

These investigators had initially tested whether a single heterozygous nonsense mutation could elicit DM1 phenotypes in mice. LoF variants, including frameshift insertion or deletion, were inducted into Dmpk, Six5, Mbnl1 and Dmwd genes in the AGhaESCs by CRISPR-Cas9 methods. None of the single-gene LoF mutants induced more than a single feature of the DM1 clinical phenotypes. However, the authors did determine for the first time the presence of muscle wasting in $D m w d^{+/-}$mice, suggesting that the Dmwd gene product is involved in the development of the DM1 disease. Yin and coworkers then created a triple LoF mutant mouse (DSM-TKO) which contained LoF variants in the three most well-known DM1-associated genes (Dmpk, Six5, Mbnl1). The DSMTKO mice exhibit most of the typical disease manifestations of adult-onset DM1, but not congenital DM1 patterns or the development of cataracts that are part of the DM1 anomalies. These triple LoF mutants presented with myotonia, muscle wasting, muscle weakness and a similar histopathology to that of DM1 patients in terms of their skeletal muscles. This mouse also recapitulated cardiac problems, endocrine disorders, digestive system disorders and defective satellite cells. To further improve their DM1 mouse model, Yin and colleagues modified the AGhaESC cells to include a LoF variant in the fourth DM1-related gene $(D m w d)$, and they referred to this as the quadruple mutant mouse model DSMD-QKO (Fig. 1). These mice expressed all of the known clinical phenotypes of the typical DM1 patient, including the battery of congenital myotonic dystrophy symptoms such as hypotonia, respiratory problems and developmental delay. The off-target problems often associated with the CRISPR-Cas9 technique were excluded in this study by whole-genome sequencing. Defects in muscle stem cells (MuSCs), the most recently described defects in DM1 patients, were also found in the DSM-TKO and DSMD-QKO DM1 mouse models, supporting the high quality and value of these DM1 mouse models for hypothesis-driven studies and drug screening schemes.

To demonstrate the utility of the DSMD-QKO DM1 mouse models, Yin and co-authors tested the phenotypic conservation in

\footnotetext{
${ }^{1}$ Center for Precision Environmental Health, Departments of Molecular and Cellular Biology and Medicine, Baylor College of Medicine, Houston, TX 77030, USA and ${ }^{2}$ Department of Molecular and Human Genetics, Baylor College of Medicine, Houston, TX 77030, USA

Correspondence: Richard H. Finnell (Richard.Finnell@bcm.edu)
}

Published online: 17 January 2020 
a

control
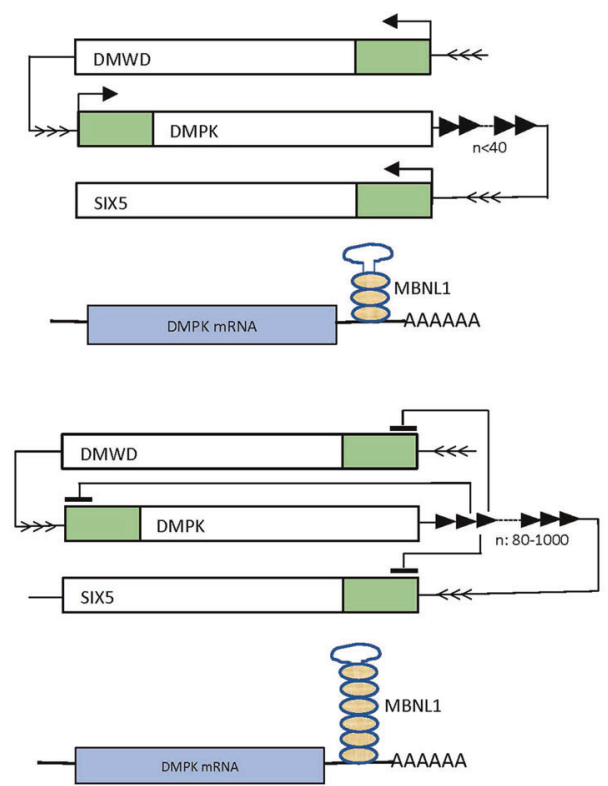

: CTG trinucleotide repeat b

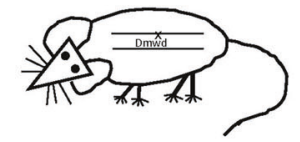

Dmwd+-SC mice with 1/9 DM1 phenotype

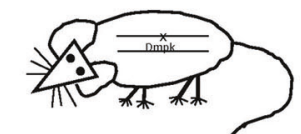

$\mathrm{Dmpk}^{+/-}$SC mice with 0/10 DM1 phenotype
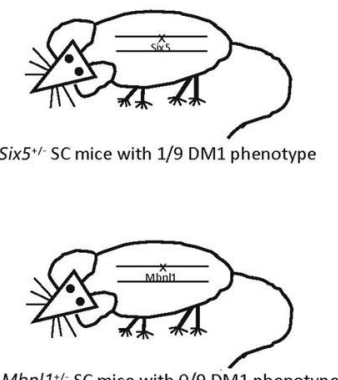

$X$ indel loss of function variant induced by CRISPR-Cas
C

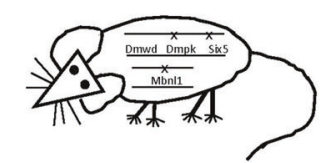

DSM-TKO SC mice with $9 / 9$ adult-onset DM1 phenotype

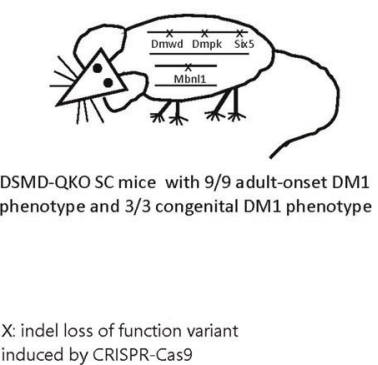

Fig. 1 DM1-related genes in control and case mice and CRISPR-Cas9 edited SC mice. a DMPK 3'-UTR CTG trinucleotide repeat expansion represses itself, upstream DMWD and downstream SIX5 gene expression, which results in the accumulation of MBNL1 in DMPK mRNA $3^{\prime}$-UTR in DM1 cases. b Single key gene heterozygous SC mice failed to recapitulate the DM1 phenotype. c TKO or QKO heterozygous SC mouse faithfully express the DM1 clinical phenotypes.

the F1 progeny of the semi-cloned (SC) mice that originated from the genetically modified AG-hsESCs. The results confirmed that F1 QKO mice maintained the typical phenotypes observed in the SC mice including splicing problems, muscle structural organization and fiber size defects, and a high percentage of central nuclei in the tibialis anterior muscles. Additionally, they investigated the usefulness of the TKO and DKO models in small-molecule screens, which is usually the first step in drug development protocols. A high-throughput screening of 10,000 compounds identified 17 positive hits, including one compound that was demonstrated to specifically rescue the differentiation defects of TKO MuSCs in vitro. These assays confirmed that the TKO and QKO mouse models are highly useful tools for potential DM1 drug development.

This study represents an excellent proof of principle demonstrating that the combination of CRISPR-Cas9 and AG-haESC could be an extremely powerful tool for studying multigenetic and/or multisystem complex diseases including birth defects (congenital heart and neural tube defects), aging-related disorders, cardiovascular diseases, cancers and neurophysiological diseases. With the growth of next generation sequencing, large numbers of diseases are now known to be caused by multigenic functional variants or diseases that can be rescued by the interaction of selected genetic variants. To efficiently make mouse models carrying the same multigenic variants in a timely and costeffective manner, investigators would be wise to replicate the same technical approaches demonstrated by Yin and colleagues in their landmark publication. This approach is vital for dissecting out the underlying mechanisms and identifying potential treatment or rescue interventions for these clinically significant and challenging diseases.

\section{REFERENCES}

1. Meola, G. \& Cardani, R. Biochim. Biophys. Acta 1852, 594-606 (2015).

2. Brook, J. D. et al. Cell 68, 799-808 (1992).

3. Mahadevan, M. et al. Science 255, 1253-1255 (1992).

4. Ricker, K. et al. Neurology 44, 1448-1452 (1994).

5. Fu, Y. H. et al. Science 260, 235-238 (1993).

6. Philips, A. V., Timchenko, L. T. \& Cooper, T. A. Science 280, 737-741 (1998).

7. Klesert, T. R., Otten, A. D., Bird, T. D. \& Tapscott, S. J. Nat. Genet. 16, 402-406 (1997).

8. Alwazzan, M., Newman, E., Hamshere, M. G. \& Brook, J. D. Hum. Mol. Genet. 8, 1491-1497 (1999).

9. Yin, Q. et al. Cell Res. https://doi.org/10.1038/s41422-019-0264-2 (2019). 